\title{
Analysis of fluid substitution in a porous and fractured medium
}

\author{
Samik Sil ${ }^{1}$, Mrinal K. Sen ${ }^{2}$, and Boris Gurevich ${ }^{3}$
}

\begin{abstract}
To improve quantitative interpretation of seismic data, we analyze the effect of fluid substitution in a porous and fractured medium on elastic properties and reflection coefficients. This analysis uses closed-form expressions suitable for fluid substitution in transversely isotropic media with a horizontal symmetry axis (HTI). For the HTI medium, the effect of changing porosity and water saturation on (1) P-wave moduli, (2) horizontal and vertical velocities, (3) anisotropic parameters, and (4) reflection coefficients are examined. The effects of fracture density on these four parameters are also studied. For the model used in this study, a $35 \%$ increase in porosity lowers the value of P-wave moduli by maximum of $45 \%$. Consistent with the reduction in $\mathrm{P}$-wave moduli, $\mathrm{P}$-wave velocities also decrease by maximum of $17 \%$ with a similar increment in porosity. The reduction is always larger for the horizontal P-wave modulus than for the
\end{abstract}

vertical one and is nearly independent of fracture density. The magnitude of the anisotropic parameters of the fractured medium also changes with increased porosity depending on the changes in the value of P-wave moduli. The reflection coefficients at an interface of the fractured medium with an isotropic medium change in accordance with the above observations and lead to an increase in anisotropic amplitude variation with offset (AVO) gradient with porosity. Additionally, we observe a maximum increase in P-wave modulus and velocity by $30 \%$ and $8 \%$, respectively, with a $100 \%$ increase in water saturation. Water saturation also changes the anisotropic parameters and reflection coefficients. Increase in water saturation considerably increases the magnitude of the anisotropic AVO gradient irrespective of fracture density. From this study, we conclude that porosity and water saturation have a significant impact on the four studied parameters and the impacts are seismically detectable.

\section{INTRODUCTION}

Fluid substitution is important for understanding in situ hydrocarbon saturation in time-lapse seismic studies, carbon dioxide $\left(\mathrm{CO}_{2}\right)$ sequestration, and many other geophysical applications. To date, most of the case studies on fluid substitution use isotropic earth models. On the other hand, most land reservoirs are fractured (hence, seismically anisotropic) to some extent. Therefore, by using anisotropic fluid substitution, we can expect to improve the results of geophysical applications. Anisotropic fluid substitution may also help to perform better seismic fracture characterization.

Many fractured reservoirs such as carbonates and tight sands contain two types of voids: (1) stiff voids (e.g., pores or vugs), also known as equant porosity, which contain most of the hydrocarbons; and (2) fractures, the volume of which is generally small compared with the overall volume of the rock, and they act as permeability conduits (Thomsen, 1995; Nelson, 2001; Neves et al., 2003; Holditch, 2006; Gurevich et al., 2009). As the simplest scenario, we consider a system of vertical fractures embedded in the reservoir matrix and aligned along a preferred horizontal direction. We can add further simplification by considering that fractures are hydraulically isolated from each other (but not from pores) and rotationally symmetric about the fracture normal. This alignment allows the medium to be treated as transversely isotropic with a horizontal axis of symmetry (HTI). In such an HTI medium, the effect of waveinduced fluid flow on the elastic properties of the rock is, in general, frequency dependent (Galvin et al., 2007). On the other hand, at low frequencies, fluid pressures have sufficient time to equilibrate between pores and fractures, and theoretical models for fractures in nonporous elastic media are not applicable (Thomsen, 1995, Hudson et al., 2001). Seismic frequency is usually (but not always) below the characteristic frequencies of a porous and fractured rock system. Therefore, low-frequency fluid substitution theories should be

Manuscript received by the Editor 5 July 2010; revised manuscript received 12 January 2011; published online 23 May 2011.

${ }^{1}$ ConocoPhillips Company, Houston, TX. E-mail: samiksil@gmail.com.

${ }^{2}$ University of Texas at Austin, Institute for Geophysics, Austin, TX.

${ }^{3}$ Curtin University, Department of Exploration Geophysics, Perth, Australia and CSIRO Earth Science and Resource Engineering, Perth, Australia. (C) 2011 Society of Exploration Geophysicists. All rights reserved. 
adequate to explain seismically observable anisotropic property changes in the fractured media that are due to fluid flow.

Thomsen (1995) quantified the fluid substitution effect in a lowfrequency limit using penny-shaped cracks and a spherical equant pores model. Hall and Kendall (2000) have used a similar model to predict the fluid-substituted response of a fractured reservoir. These works are limited by considering fracture geometry as pennyshaped cracks. More recently, Cardona (2002a) and Gurevich (2003) presented a fluid substitution scheme independent of fracture geometry using anisotropic Gassmann (1951) and Brown and Korringa (1975) equations. Gurevich (2003) further developed explicit analytical expressions for fluid substitution particularly applicable in a porous HTI medium. These equations are relatively easy to implement and are free from any assumption on the extent of fracturing. Using Gurevich's (2003) HTI fluid substitution equations, we attempt to determine the effect of changing background porosity and water saturation of a porous HTI medium on (1) P-wave moduli (anisotropic), (2) P-wave velocities (vertical $V_{P}^{v}$ and horizontal $V_{P}^{h}$ ), (3) "Thomsen-style" anisotropic parameters (Thomsen, 1986; Rüger, 1997), and (4) reflection coefficients. The effect of changing fracture density on the above four parameters is also studied. Note that according to the linear slip theory (Schoenberg and Sayers, 1995), porosity and fluid saturation (cases in which fracture porosity is very small) are related to the properties of the background isotropic medium. Therefore, the effects of changes in properties of the background isotropic material on the effective anisotropic properties of a medium may not be obvious or expected. The primary goal of this paper is to document the changes in the seismic-anisotropy-related reservoir model properties that are due to fluid substitution. We also comment on the magnitude of those changes.

To perform the analysis, we use a two-layered, half-space model. The top layer is assumed to be shale (seal of the reservoir) and the bottom layer an HTI sandstone reservoir. Generally, shale is seismically anisotropic, but for the simplification of our analysis, we assume an isotropic overburden model. We further assume availability of all of the necessary rock physics parameters (isotropic solid background and fluid properties) for the HTI medium fluid substitution (e.g., for the reservoir we use average properties of a high porosity sandstone from Mavko et al., 2003, and fluid properties from the same source). For the overburden shale (only used to calculate reflection coefficients), we use a P-wave velocity of $3.85 \mathrm{~km} / \mathrm{s}$, an S-wave velocity of $2.15 \mathrm{~km} / \mathrm{s}$, and a density of $2.5 \mathrm{gm} / \mathrm{cc}$ (all of these values are consistent with the shale properties from Castagna et al., 1993). We do not consider any porosity in the shale and avoid fluid substitution in it. We then compute the changes in $\mathrm{P}$-wave moduli $\left(C_{11}\right.$ and $\left.C_{33}\right)$, $\mathrm{P}$-wave velocities, and anisotropy parameters of the sandstone reservoir with porosity and water saturation. We also compute the reflection coefficient changes between the shale-sandstone unit for the porosity and water saturation changes. Our analysis suggests that conspicuous changes in the anisotropic sandstone properties should be manifested by fluid substitution and porosity changes.

\section{THEORY}

\section{Anisotropic fluid substitution using Gassmann equations}

Gurevich et al. (2009) presented a detailed overview of anisotropic fluid substitution using Gassmann (1951) equations. Here we briefly review the discussion on anisotropic Gassmann equa- tions for the completeness of this paper. In seismic analysis, isotropic Gassmann (1951) equations are routinely used to model the fluid effect (Mavko et al., 2003; Smith et al. 2003). According to these equations, the bulk modulus of an isotropic fluid-saturated porous rock $\left(K^{\text {sat }}\right)$ can be obtained by summing the dry bulk modulus of the rock $(K)$ and an additional fluid effect term

$$
K^{\text {sat }}=K+\alpha^{2} M
$$

In equation $1, \alpha=1-K / K_{g}$ represents the Biot-Willis (Biot and Willis, 1957) coefficients ( $K_{g}$ is the solid grain bulk modulus), and

$$
M=\frac{K_{g}}{\left(1-\frac{K}{K_{g}}\right)-\phi\left(1-\frac{K_{g}}{K_{f}}\right)},
$$

is the pore-space modulus, $\varphi$ is the porosity, and $K_{f}$ is the fluid bulk modulus. According to this theory, dry and fluid-saturated shear modulus values are same (i.e., $\mu=\mu^{\text {sat }}$ ).

Along with the isotropic cases, Gassmann (1951) also presented similar equations for fluid substitution in an anisotropic medium (applicable to the low-frequency limit). However, for the anisotropic case, instead of using the bulk modulus, Gassmann expressed a saturated stiffness matrix of the anisotropic rock by summing the dry stiffness matrix and an additional fluid effect term:

$$
C_{i j}^{s a t}=C_{i j}^{d r y}+\alpha_{i} \alpha_{j} M . \quad i, j=1, \ldots 6 .
$$

Here, $C_{i j}^{d r y}$ is the dry frame stiffness matrix of the anisotropic medium and

$$
\alpha_{m}=1-\frac{\sum_{n=1}^{3} C_{n m}^{d r y}}{3 K_{g}}
$$

for $m=1,2$, and 3, and $\alpha_{4}=\alpha_{5}=\alpha_{6}=0$. Note that when the medium is isotropic, from equation 4 we get $\alpha_{\mathrm{m}}=\alpha=1-K / K_{\mathrm{g}}$. Similarly, $M$ in equation 3 is the direct analogue of the isotropic pore space modulus (as present in equation 2) and can be expressed as

$$
M=\frac{K_{g}}{\left(1-\frac{K^{*}}{K_{g}}\right)-\varphi\left(1-\frac{K_{g}}{K_{f}}\right)},
$$

with

$$
K^{*}=\frac{1}{9} \sum_{i=1}^{3} \sum_{j=1}^{3} C_{i j}^{d r y},
$$

where $K^{*}$ is known as the effective bulk modulus. Once again, note that when the medium is isotropic, $K^{*}$ in equation 6 reduces to $K$ and therefore equation 5 reduces to equation 2. Equations 3-6 help us to perform fluid substitution in an anisotropic medium when dry stiffness values of the anisotropic medium and background solid (matrix) and fluid properties are known.

\section{Porous HTI medium}

Gurevich (2003) derived explicit expressions for fluid substitution in porous HTI medium based on Gassmann's anisotropic fluid substitution equations. In an HTI medium fractures are 
aligned along a preferred direction (perpendicular to the symmetry axis of the HTI medium, Figure 1). For such an HTI medium, expression for the dry stiffness matrix can be obtained from Schoenberg and Sayers (1995) as

$C_{i j}^{d r y}(H T I)=\left[\begin{array}{cccccc}M_{b}\left(1-\Delta_{N}\right) & \lambda\left(1-\Delta_{N}\right) & \lambda\left(1-\Delta_{N}\right) & 0 & 0 & 0 \\ \lambda\left(1-\Delta_{N}\right) & M_{b}\left(1-r^{2} \Delta_{N}\right) & \lambda\left(1-r \Delta_{N}\right) & 0 & 0 & 0 \\ \lambda\left(1-\Delta_{N}\right) & \lambda\left(1-r \Delta_{N}\right) & M_{b}\left(1-r^{2} \Delta_{N}\right) & 0 & 0 & 0 \\ 0 & 0 & 0 & \mu & 0 & 0 \\ 0 & 0 & 0 & 0 & \mu\left(1-\Delta_{T}\right) & 0 \\ 0 & 0 & 0 & 0 & 0 & \mu\left(1-\Delta_{T}\right)\end{array}\right]$.

Here

$$
\begin{gathered}
M_{b}=\lambda+2 \mu, \\
r=\frac{\lambda}{M_{b}}, \\
\Delta_{N}=\frac{Z_{N} M_{b}}{1+Z_{N} M_{b}},
\end{gathered}
$$

and,

$$
\Delta_{T}=\frac{Z_{T} \mu}{1+Z_{T} \mu} .
$$

In the above equations, $\lambda$ and $\mu$ are the Lame parameter and shear modulus of the background isotropic material, respectively, and $Z_{N}$ and $Z_{T}$ are the normal and tangential fracture compliances of the HTI medium, respectively. $\Delta_{N}$ and $\Delta_{T}$ of equations 10 and 11 are the normal and tangential fracture weaknesses of the medium, respectively (Schoenberg and Sayers, 1995).

Now, using dry $C_{i j}$ values of the HTI medium from expression 7 and using equations 4 and 6 , we have

$$
\begin{gathered}
\alpha_{1}=\alpha_{0}+\frac{K \Delta_{N}}{K_{g}}, \\
\alpha_{2}=\alpha_{3}=\alpha_{0}+\frac{K \lambda \Delta_{N}}{K_{g}(\lambda+2 \mu)},
\end{gathered}
$$

and

$$
K^{*}=K\left(1-\frac{K \Delta_{N}}{\lambda+2 \mu}\right)
$$

Other parameters $\alpha_{4}, \alpha_{5}$, and $\alpha_{6}$ are equal to zero for the HTI medium (like any other anisotropic medium). In these equations, $K=\lambda+2 / 3 \mu$ is the dry bulk modulus of the background isotropic rock and $\alpha_{0}=1-K / K_{g}$ (similar to isotropic medium $\alpha$ or Biot-Willis coefficients). Equation 14 indicates that the effective bulk modulus of the HTI medium is lower than the bulk modulus of the isotropic background medium because of the presence of the fractures.

Equations 3, 5, and 12-14 can be used for porous HTI medium fluid substitution. They can also be used to obtain individual fluid substitution expressions for each stiffness element of the HTI medium:

$$
C_{11}^{S a t}=\frac{L}{D}\left\{d_{1} \theta+\frac{K_{F}}{\varphi K_{g} L}\left[L_{1} \alpha^{\prime}-\frac{16 \mu^{2} \alpha_{0} \Delta_{N}}{9 L}\right]\right\},
$$

$$
\begin{gathered}
C_{33}^{S a t}=\frac{L}{D}\left\{d_{2} \theta+\frac{K_{F}}{\varphi K_{g} L}\left[L_{1} \alpha^{\prime}-\frac{4 \mu^{2} \alpha_{0} \Delta_{N}}{9 L}\right]\right\}, \\
C_{13}^{S a t}=\frac{\lambda}{D}\left\{d_{1} \theta+\frac{K_{F}}{\varphi K_{g} \lambda}\left[\lambda_{1} \alpha^{\prime}+\frac{8 \mu^{2} \alpha_{0} \Delta_{N}}{9 L}\right]\right\}, \\
C_{44}^{\text {Sat }}=\mu,
\end{gathered}
$$

and

$$
C_{55}^{S a t}=\mu\left(1-\Delta_{T}\right) .
$$

Accurate expressions for the parameters such as $L, L_{1}, D, d_{1}, d_{2}$, $\alpha_{0}, \alpha^{\prime}$, and $\lambda_{1}$ used in equations 15-19 can be found in Gurevich (2003) and Brown and Gurevich (2004). We present those expressions below for the completeness of this paper:

$$
\begin{gathered}
L=\lambda+2 \mu, \\
D=1+\frac{K_{F}}{K_{g} \phi}\left(\alpha_{0}-\phi+\frac{K^{2} \Delta_{N}}{K_{g} L}\right),
\end{gathered}
$$

$$
\begin{gathered}
\theta=1-\frac{K_{F}}{K_{g}}, \\
\alpha_{o}=1-\frac{K}{K_{g}}, \\
\alpha^{\prime}=\alpha_{0}+\frac{K^{2}}{K_{g} L} \Delta_{N}, \\
L_{1}=K_{g}+\frac{4}{3} \mu,
\end{gathered}
$$

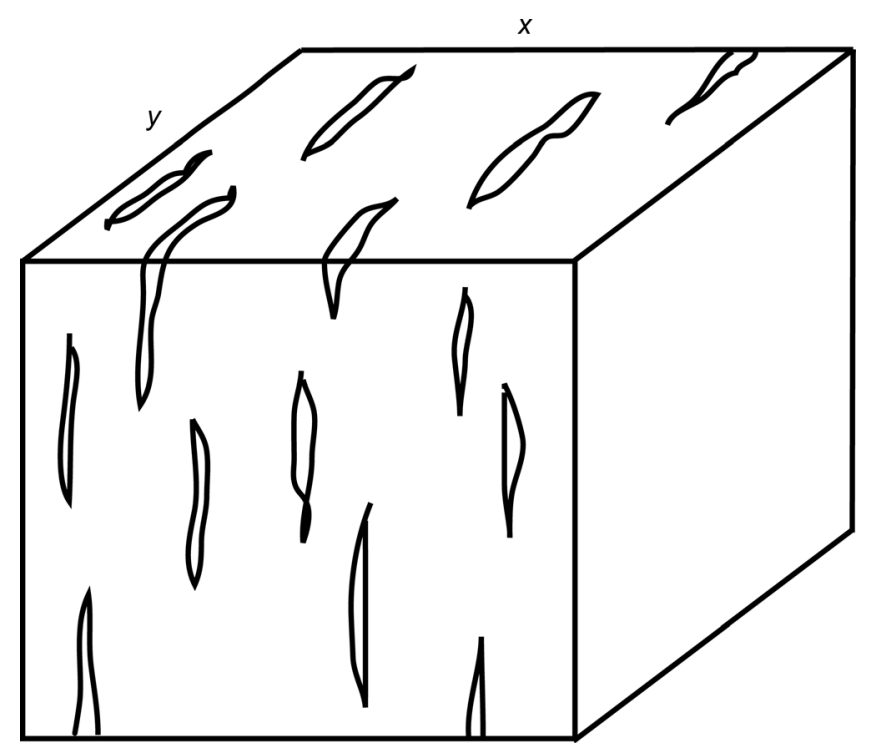

Figure 1. A cartoon representation of an HTI medium. Fracture strike directions are uniform (along $y$-direction). Symmetry axis of the medium is along the $x$-direction. 


$$
\begin{aligned}
& \lambda_{1}=K_{g}-\frac{2}{3} \mu \\
& d_{1}=1-\Delta_{N},
\end{aligned}
$$

and

$$
d_{2}=1-\frac{\lambda^{2}}{L^{2}} \Delta_{N} .
$$

In the work presented here, equations 15-19 (along with equations 20-28) are used in the fluid substitution analysis for the porous HTI medium.

\section{SYNTHETIC EXAMPLES AND RESULTS}

Our goal of this study is to analyze the effect of fluid substitution in a porous and fractured (HTI) medium. For the purpose of the investigation, we consider a fractured sand model with a background dry P-wave velocity of $3.8 \mathrm{~km} / \mathrm{s}$, a dry S-wave velocity of $2.16 \mathrm{~km} / \mathrm{s}$, a density of $2.65 \mathrm{gm} / \mathrm{cc}$ (grain density), and
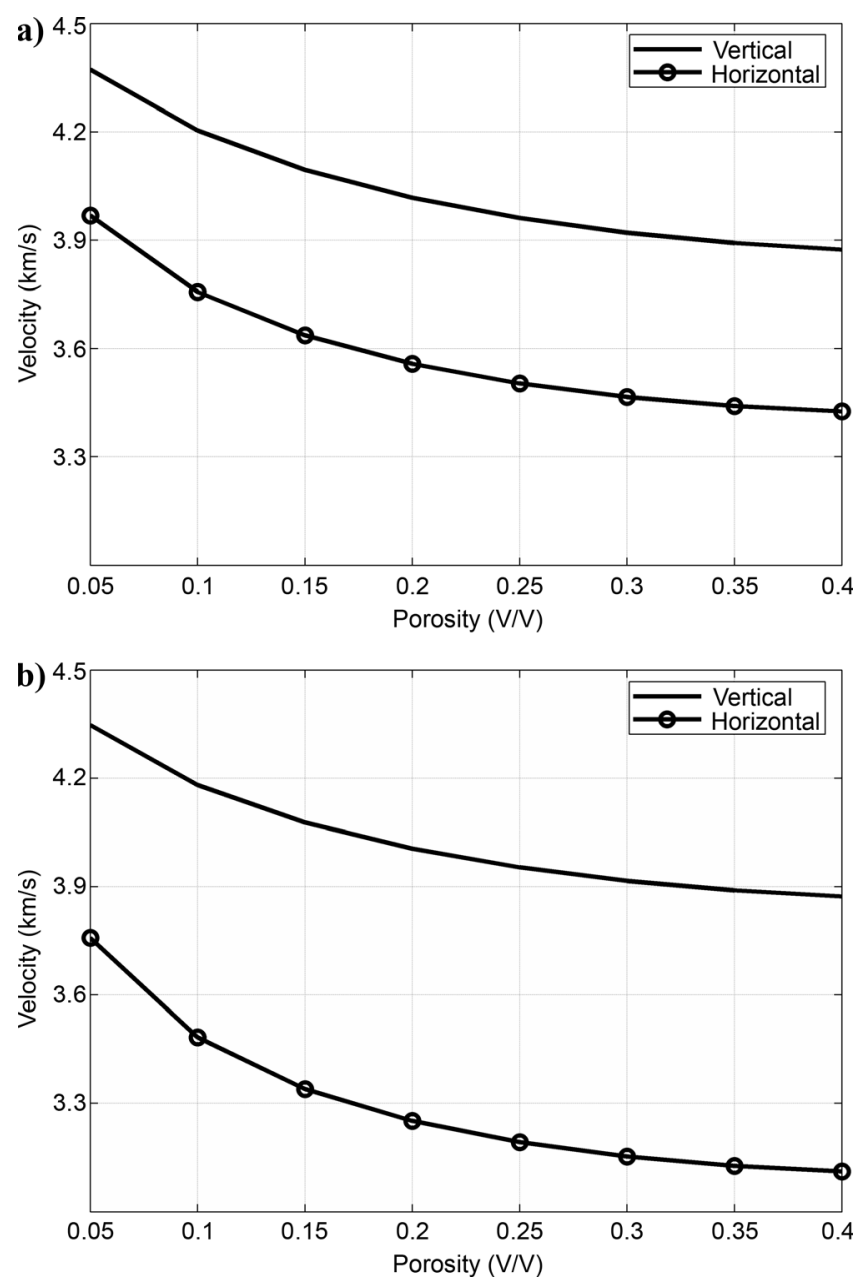

Figure 2. Variation of P-wave velocities with changing porosity (a) for low fracture density of 0.08 and (b) for higher fracture density of 0.16 . a high-porosity sandstone as documented by Mavko et al. (2003). We then add extra fracture compliances $\left(Z_{T}\right.$ and $\left.Z_{N}\right)$ to the isotropic background properties to make the medium HTI. We consider two cases: case 1 for low fracture density $(0.08)$ and case 2 for high fracture density (0.16). Fracture density $(F D)$ is calculated using the following equation (Cardona, 2002b):

$$
F D=\frac{3 Z_{T} \mu(2-v)}{16(1-v)}
$$

Here, $v$ is Poisson's ratio of the background isotropic medium. The sandstone layer is assigned $20 \%$ porosity.

We also study the effect of fluid substitution on reflection coefficients. For this purpose, we introduce an isotropic top layer over the target HTI model. We assign the top layer the properties of shale with a P-wave velocity of $3.85 \mathrm{~km} / \mathrm{s}$, an S-wave velocity of $2.15 \mathrm{~km} / \mathrm{s}$, and a density of $2.5 \mathrm{gm} / \mathrm{cc}$. These values are consistent with the shale properties and can be explained by Gardner's relation (Castagna et al., 1993). In general, shale is seismically anisotropic in nature. Nevertheless, because our work focuses on the fluid substitution in fractured sand, we consider isotropic overburden for the purpose of simplification of the reflection coefficient results (isotropicanisotropic interface case). We do not consider any porosity (or fluid substitution effect) on the top shale layer.

With the above modeling setup and using equations 15-19, we study the effect of porosity on P-wave moduli, P-wave velocities, and anisotropic parameters for $100 \%$ water-saturated sand. First, we analyze the variation of horizontal and vertical P-wave moduli ( $C_{11}$ and $C_{33}$ respectively) with porosity for case 1 (fracture density 0.08 ) and case 2 (fracture density is doubled to 0.16). In both of the cases, we observe a decrease in the P-wave moduli as porosity increases. We observe a $37 \%$ decrease in $C_{33}$ and a $41 \%$ decrease in $C_{11}$ with an increase of $35 \%$ in porosity for case 1 . For case 2 , with a similar increase in porosity, we observe a $37 \%$ decrease in $C_{33}$ and a $45 \%$ decrease in $C_{11}$. An increase in the fracture density causes an overall decrease in the initial value of the moduli (more decrement for the $C_{11}$ or horizontal component). However, on average, the effect of porosity remains the same for case 1 and case 2 .

Figure $2 \mathrm{a}$ shows the effect of changing porosity on vertical and horizontal $\mathrm{P}$-wave velocities for lower fracture density (case 1), and Figure $2 \mathrm{~b}$ shows a similar plot for the higher fracture density medium (case 2). In both cases, just like P-wave moduli, P-wave velocities tend to decrease with increasing porosity. Similar to P-wave moduli, increasing fracture density also lowers the initial value of $\mathrm{P}$-wave velocities by making the medium more compliant. The effect is more dominant on the horizontal velocity than on the vertical velocity. But, with increasing porosity, we observe a similar decrease in horizontal and vertical velocities for case 1 and case 2 (11\% decrease for the vertical velocities with porosity in case 1 and case $2 ; 14 \%$ and $17 \%$ decrease in horizontal velocities for case 1 and case 2, respectively). The above observations may suggest that the effect of porosity is the dominant factor in the decrement rate of the P-wave velocities with porosity compared with the effect of fracture density. 
Figure 3a shows the variation of three anisotropy parameters $\left(\varepsilon^{v}, \gamma^{v}\right.$, and $\left.\delta^{v}\right)$ for the HTI medium (as defined by Rüger, 1997) as a function of porosity for case 1. As expected from the theory, no change is observed in $\gamma^{v}$ with porosity. There is a decrease in $\varepsilon^{v}$ by $25 \%$ (but increase in absolute value) and a $46 \%$ increase in $\delta^{v}$ (but a decrease in absolute value). Note that the change in porosity occurs in the background isotropic medium, but that does affect the anisotropic parameters $\left(\varepsilon^{v}\right.$ and $\left.\delta^{v}\right)$ because of pressure communication between pores and fractures. In case 2, when the fracture density is higher, we observe an overall increase in magnitude of all of the anisotropic parameters (Figure $3 b$ ). However, their variation with porosity remains the same (a $30 \%$ decrease in $\varepsilon^{v}$ and a $41 \%$ increase in $\delta^{v}$ with increasing porosity).
Figure 4 illustrates the effect of matrix porosity on azimutal variation of reflection coefficients for the isotropic and HTI medium. The left panel of Figure $4 \mathrm{a}$ shows the plot of reflection coefficients as functions of azimuth for different incident angles. A porosity of $5 \%$ is considered in this case, and the sand is assumed to be $100 \%$ water saturated. The right panel of Figure 4a shows a similar plot for a porosity of $35 \%$. Both of the plots are for case 1 (low fracture density). Sinusoidal variation of reflection coefficients with azimuth (Mallick et al., 1998) due to anisotropy is visible even at a lower incidence angle for both of the cases. The sinusoidal variation is stronger for the $35 \%$ porosity case than for the $5 \%$ porosity case. By fitting a cosine function (Sil et al., 2010), we observe an anisotropic gradient $\left(B_{A N I}\right)$ of -0.01 and -0.002 for the $35 \%$ porosity and $5 \%$

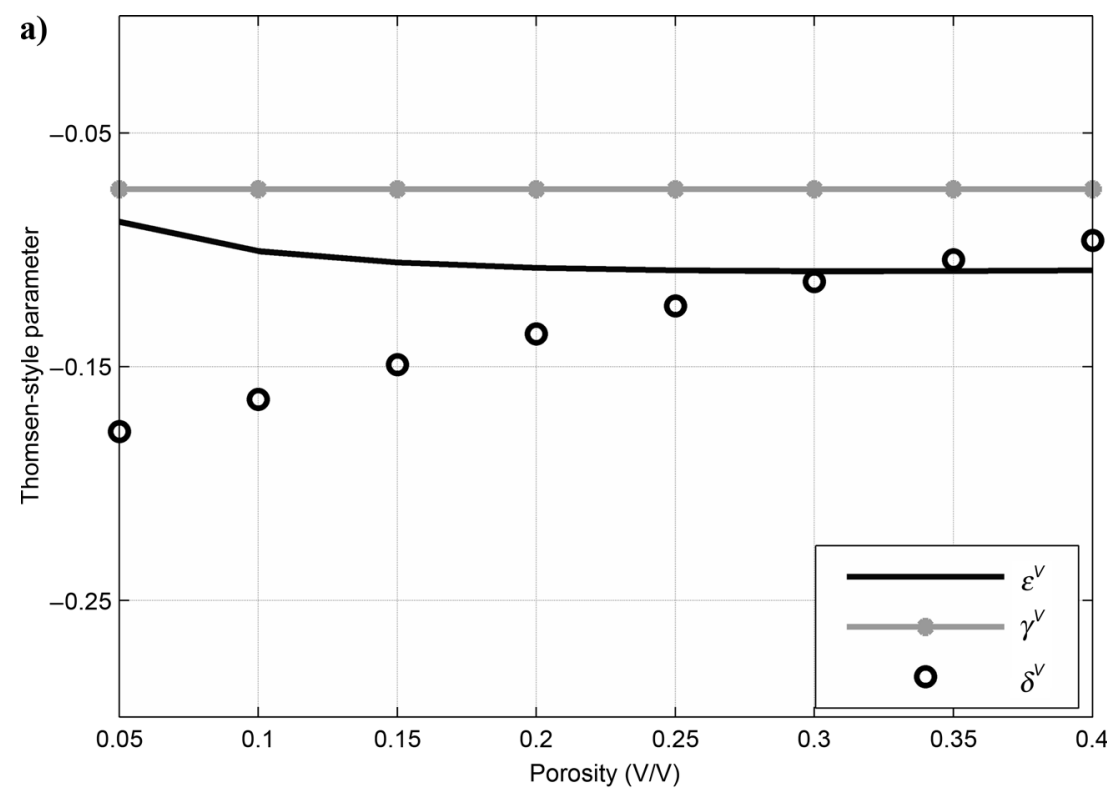

Figure 3. Variation of anisotropic parameters with changing porosity (a) for low fracture density of 0.08 and (b) for higher fracture density of 0.16 .

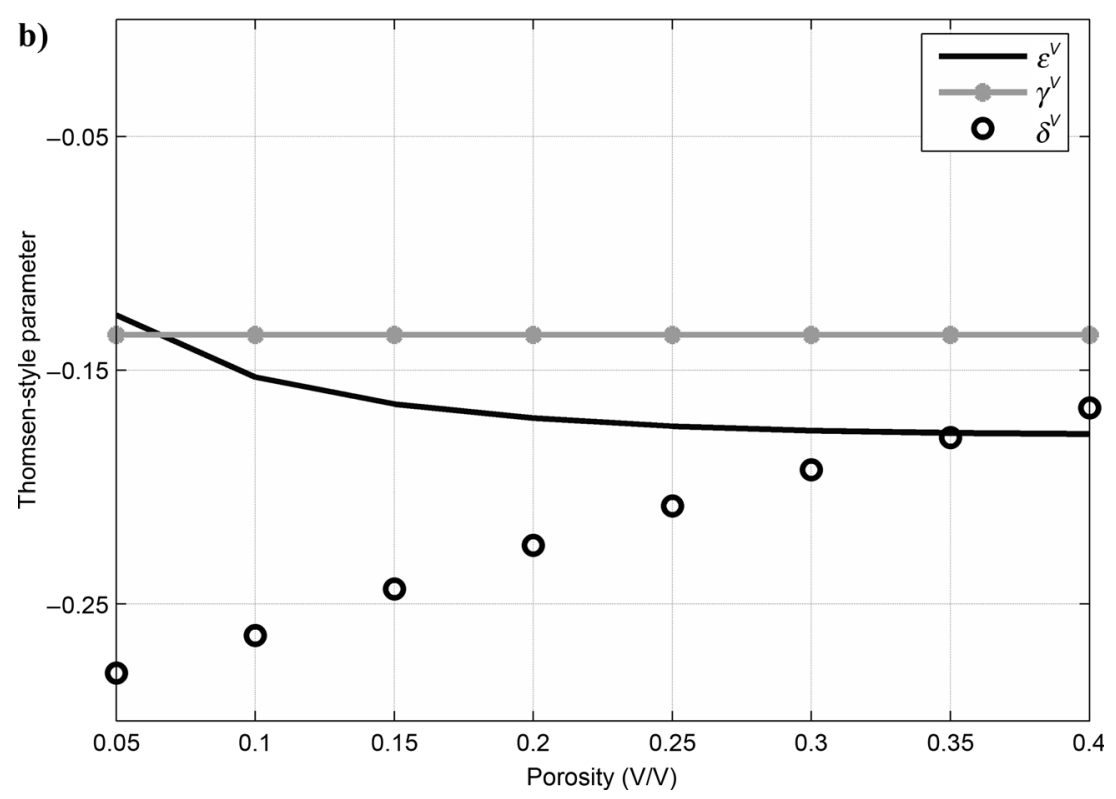


porosity cases, respectively. This implies that increasing porosity increases the azimuthal anisotropic variation in reflection coefficients. An increase of porosity changes the sign of the reflection coefficients from positive to negative. Figure $4 b$ shows a similar plot for the higher fracture density case. Here, we also observe an increase in azimuthal variation with an increase in porosity (anisotropic gradient -0.02 for higher porosity and -0.01 for lower porosity: right and left panel of Figure $4 b$, respectively.) Similar to case 1 , reflection coefficients also change sign because of an increase in porosity in case 2 . We present our observations on the effect of changing porosity in Pwave moduli, P-wave velocity, anisotropic parameters, and reflection coefficients in Table 1.
The next investigation studies the influence of water saturation $\left(S_{W}\right)$ and fracture density on P-wave moduli, P-wave velocity, and anisotropy parameters. First, we analyze the P-wave moduli with the variation of water saturation. This analysis is based on $20 \%$ porosity of the background isotropic material and lower fracture density (case 1). An increase in water saturation causes an increase in the value of P-wave moduli similar to any isotropic case. Similar analysis is performed for the higher fracture density case (case 2). Increasing the overall fracture density lowers the initial P-wave moduli values. However, that influence is more pronounced for the horizontal $C_{11}$ than for the vertical $C_{33}$. We observe an increase in the $C_{11}$ value by $20 \%$ in case 1 and $30 \%$ in case 2 with a $100 \%$ increase in water saturation. An
Figure 4. Variation of reflection coefficients with incident angle (index) and azimuth (a) for low-fracture-density case (fracture density 0.08 ). Left panel shows reflection coefficient variation for $5 \%$ porosity. Right panel shows the variation of reflection coefficients for the $35 \%$ porosity case. (b) Similar plot for higher fracture density of 0.16 .
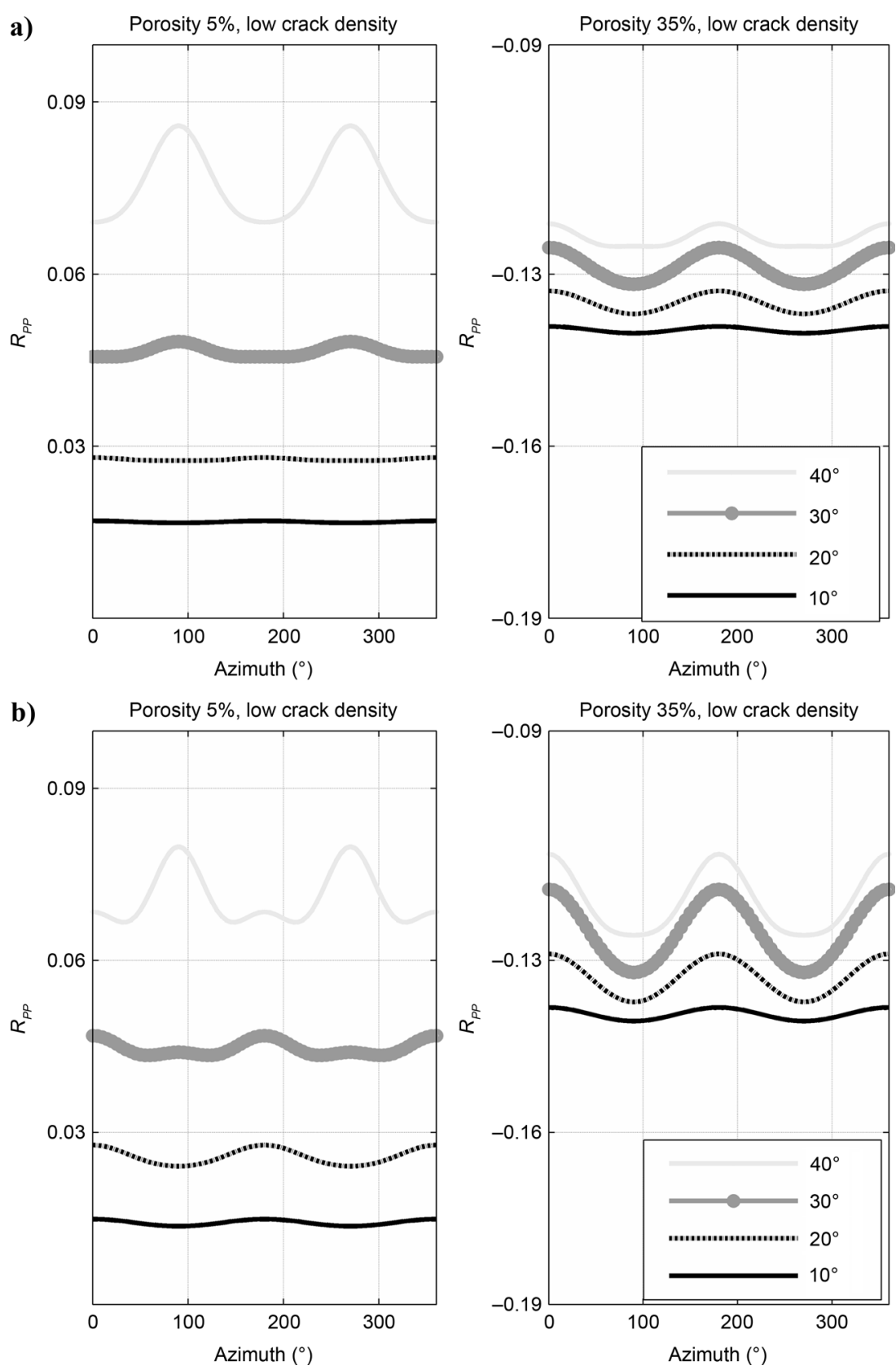
increase of $C_{33}$ values by $8 \%$ and $10 \%$ is observed with a similar increment in water saturation for case 1 and case 2, respectively.

Similar observations can be made from the plot of P-wave velocities versus water saturation in low-fracture-density (Figure 5a) and high-fracture-density cases (Figure 5b). Note that in all of the above cases, a further increase (different from the normal linear incremental trend) is observed in velocity and $\mathrm{P}$-wave moduli after $90 \%$ water saturation. In general, we observe that an increase in fracture density lowers the initial P-wave velocities. The reduction is greater for horizontal velocity than the vertical velocity. With a $100 \%$ increase in water saturation, vertical velocity increases by $0.8 \%$ and $1.5 \%$ for case 1 and case 2 , respectively. A similar increment in water saturation increases the horizontal velocity by $4.5 \%$ and $8 \%$ for case 1 and case 2, respectively.

Figure 6 shows the variation of anisotropic parameters with changes in water saturations and fracture density. No changes are observed in $\gamma^{v}$ with water saturation for case 1 (Figure 6a) and case 2 (Figure 6b). A slight increase in the magnitude of $\delta^{v}$ with water saturation is observed in case 2 (Figure 6b). Maximum changes are observed in the parameter $\varepsilon^{v}$. The magnitude of $\varepsilon^{v}$ decreases by almost $20 \%$ with increasing water saturation for case 1 and case 2 . Whereas for case 2 (higher fracture density) there is an overall increase in the magnitude of all of the anisotropic parameters, their relative behavior with water saturation remains the same as in the low-fracturedensity case.

Figure 7 shows the relationship between reflection coefficients and water saturation for case 1 and case 2 (fracture densities of 0.08 and 0.16 , respectively). Figure $7 \mathrm{a}$ shows plots of reflection coefficients as a function of azimuth and incident angle for a water saturation of $0 \%$ (left panel) and case 1 . The right panel shows the similar plot for the $100 \%$ water saturation scenario. In case 1 , we observe that an increase in water saturation decreases the magnitude of reflection coefficients. Azimuthal variation of reflection coefficients becomes greater for the $100 \%$ water-saturated case than the dry case. We estimate an anisotropic amplitude variation with offset (AVO) gradient and observe a higher anisotropic gradient for the watersaturated case $(-0.004)$ than the dry case $(-0.002)$. We make a similar observation for case 2 (Figure 7b). Here, anisotropic gradient also increases with increasing water saturation $(-0.01$ for $100 \%$ water saturation and -0.005 for dry). An increase in fracture density also increases the magnitude of the reflection coefficient.

We present our observations on the effect of changing water saturation in P-wave moduli, P-wave velocity, anisotropic parameters, and reflection coefficients in Table 2.

\section{DISCUSSION}

Our analysis suggests that porosity and water saturation have a significant effect on P-wave moduli and therefore on the $\mathrm{P}$-wave velocities and seismic anisotropy parameters of a porous fractured HTI medium. Changes in these properties can also significantly influence the AVAZ (amplitude variation with azimuth) signals and therefore should be seismically detectable. By analyzing our results documented in Tables 1 and 2, we can make some interesting observations.
We observe that a relatively small increase in porosity can significantly change all of the studied parameters (P-wave moduli, velocities, anisotropic parameters, and anisotropic gradient). Relative changes in those parameters are more sensitive to porosity than fracture density. From Table 1, we can see that most of the studied parameters show similar variations with porosity for case 1 (low fracture density) and case 2 (high fracture den-

Table 1. Summary of influence of porosity change $(\Delta \varphi)$ from $5 \%$ to $40 \%$ on $\mathrm{P}$-wave moduli $\left(\Delta C_{11}\right.$ and $\left.\Delta C_{33}\right)$, $\mathrm{P}$-wave velocities $\left(\Delta V_{P}^{h}\right.$ and $\left.\Delta V_{P}^{v}\right)$, anisotropic parameters $\left(\Delta \gamma^{v}, \Delta \varepsilon^{v}\right.$, and $\left.\Delta \delta^{v}\right)$, and anisotropic AVO gradient $\left(\Delta B_{A N I}\right)$

\begin{tabular}{llllllllll} 
Case & $\Delta \phi$ & $\Delta C_{11}$ & $\Delta C_{33}$ & $\Delta V_{P}^{h}$ & $\Delta V_{P}^{v}$ & $\Delta \gamma^{v}$ & $\Delta \varepsilon^{v}$ & $\Delta \delta^{v}$ & $\Delta B_{A N I}$ \\
\hline 1 & $35 \%$ & $-41 \%$ & $-37 \%$ & $-14 \%$ & $-11 \%$ & $0 \%$ & $-25 \%$ & $46 \%$ & $400 \%$ \\
2 & $35 \%$ & $-45 \%$ & $-37 \%$ & $-17 \%$ & $-11 \%$ & $0 \%$ & $-30 \%$ & $41 \%$ & $50 \%$
\end{tabular}
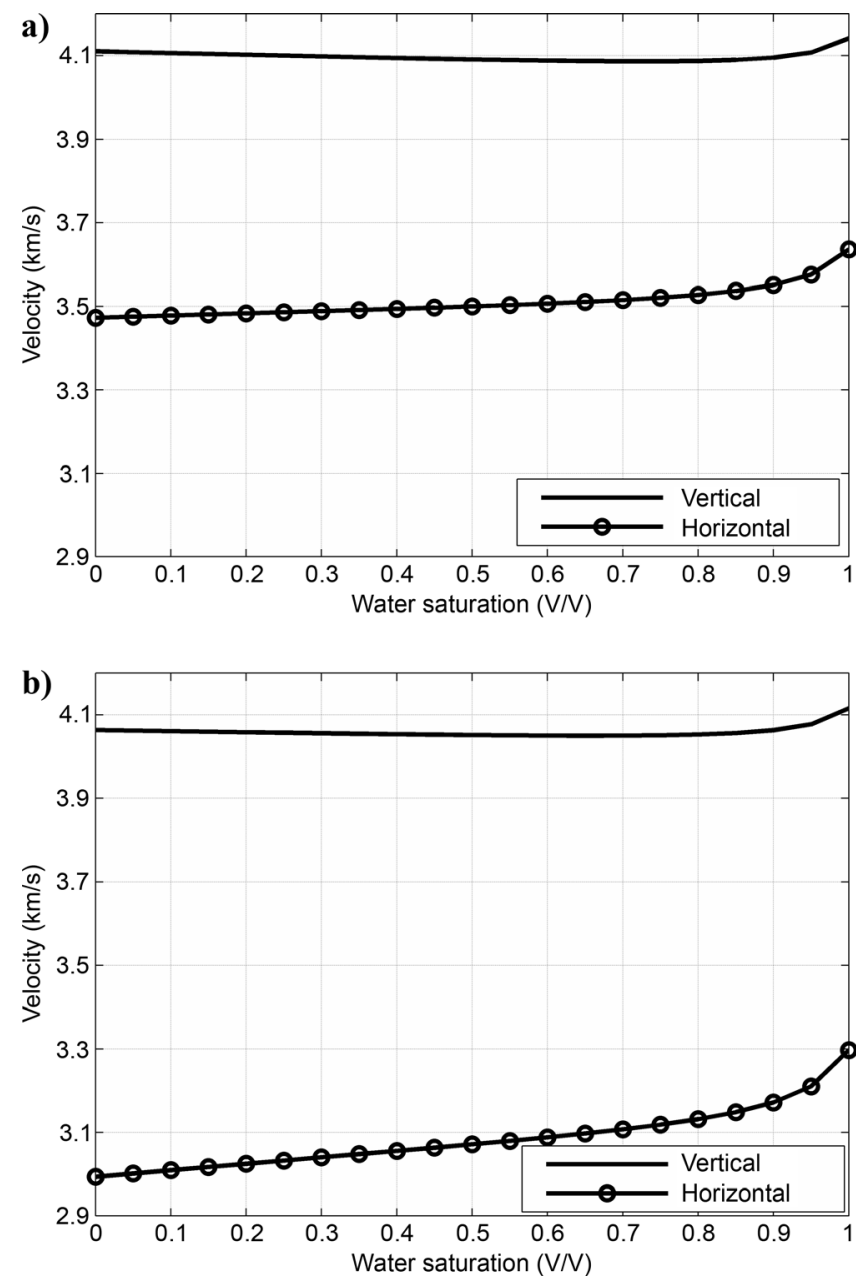

Figure 5. Variation of $\mathrm{P}$-wave velocities with changing water saturation (a) for low fracture density of 0.08 and (b) for higher fracture density of 0.16 . 
sity). This observation is interesting in the sense that a general notion about anisotropy is that its magnitude is proportional to the fracture density. In conventional AVAZ analysis, the anisotropic gradient is a measure of the fracture density. Our work suggests that such straightforward correlation between fracture density and the anisotropic gradient may result in erroneous interpretation in an area of varying porosity.

Similarly, from Table 2 we can observe that the dry and wet states of the medium have a stronger influence on the studied parameters than fracture density. Here we also can see a similar range of changes in $\mathrm{P}$-wave moduli, velocities, anisotropic parameters, and anisotropic gradient for case 1 and case 2. AVAZ signals also change significantly between the dry and wet cases.
The wet case shows higher anisotropic gradients than dry cases. Therefore, in fractured gas play areas, AVAZ signals with a higher anisotropic gradient may not be the sweet spot for production.

Porosity in the medium is considered to only be due to an isotropic background medium. Because fracture porosity is small, it is ignored in analyzing the anisotropic Gassmann equations used in this study. Similarly, water saturation also occurs mainly in the pore space of the background isotropic material of the HTI medium only. Therefore, it may not be intuitive to expect changes in the anisotropic properties with porosity and water saturation. However, our results show that changes in the porosity and water saturation in the background isotropic
Figure 6. Variation of anisotropic parameters with changing water saturation (a) for low fracture density of 0.08 and (b) for higher fracture density of 0.16 .
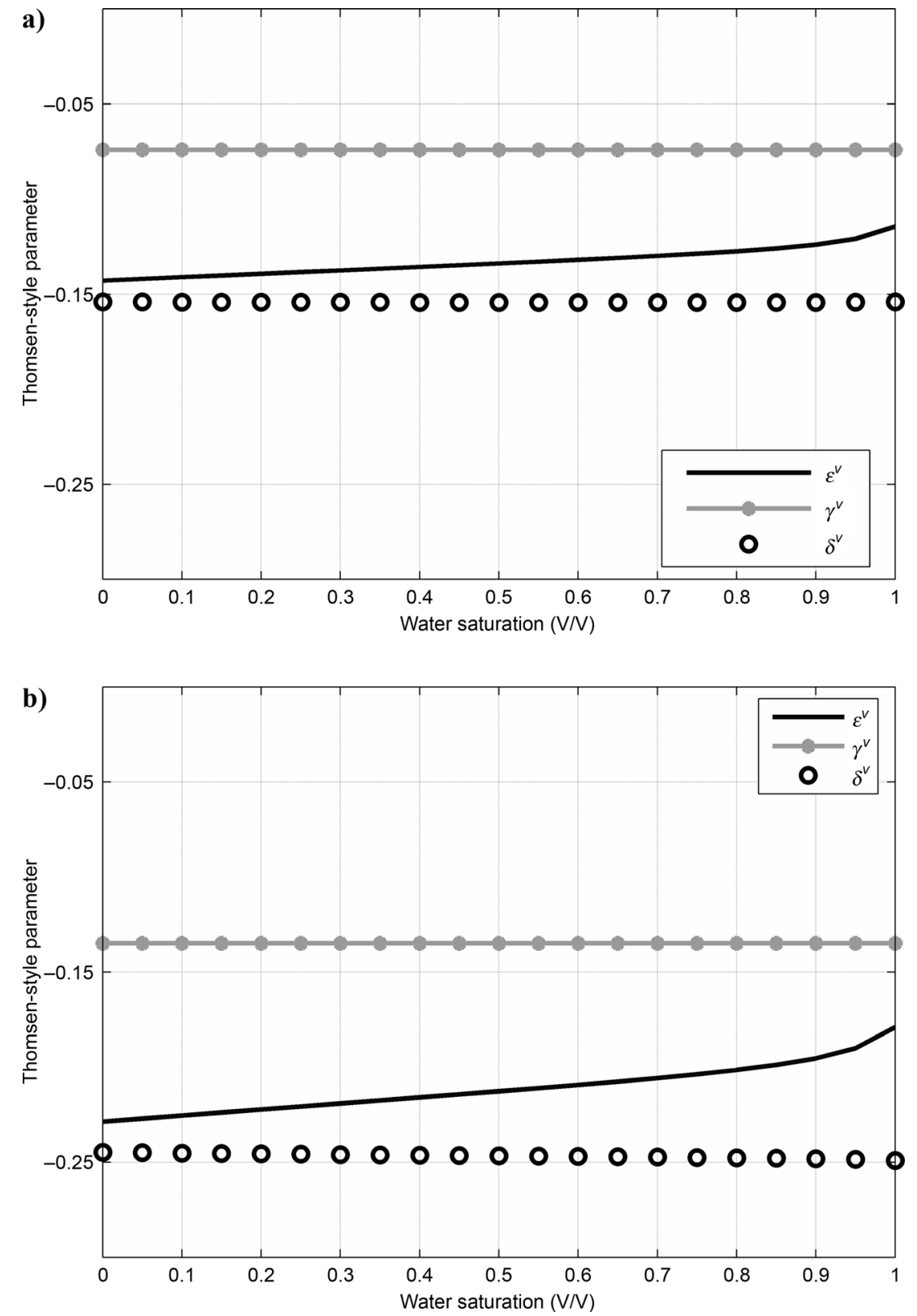
medium can affect the anisotropic parameters $\varepsilon^{v}$ and $\delta^{v}$. This observation can be explained with "linear slip theory" (Schoenberg and Sayers, 1995). According to this theory, the parameters $C_{11}$, $C_{22}, C_{13}$, and $C_{33}$ of an HTI medium contain terms $M_{b}, r$, and $\lambda$ (equations 7-9). By changing the porosity and water saturation in a medium, we also change the isotropic background property $\lambda$ (hence $M_{b}$ and $r$ ) of the medium. Hence, the observed changes in the anisotropic parameters are obvious.

There is another reason to observe changes in anisotropic parameters $\varepsilon^{v}$ and $\delta^{v}$ with changes in the background porosity and water saturation. In our model, the fracture parameters are defined in the compliance domain by additional normal frac- ture $\left(Z_{N}\right)$ compliance and tangential fracture compliance $\left(Z_{T}\right)$ terms. We interrelate these terms using Sayers and Kachanov's (1995) formula to maintain consistency of the model. The formula is

$$
\frac{Z_{N}}{Z_{T}}=1-v / 2
$$

where $v$ is Poisson's ratio of the background isotropic medium. With changing porosity and water saturation, the value of background $v$ also changes accordingly. Therefore the observed changes in the anisotropic parameters with changing background porosity and water saturation are not surprising.
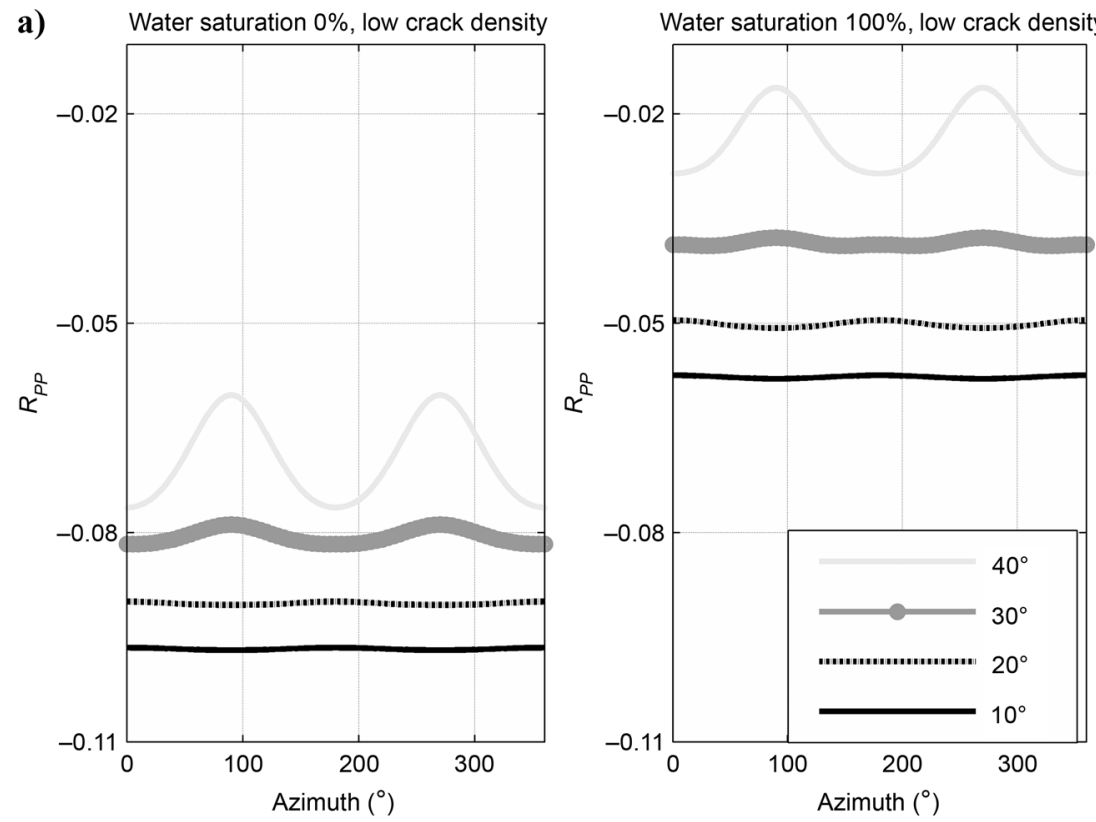

Figure 7. Variation of reflection coefficients with incident angle (index) and azimuth (a) for low-fracture-density case (fracture density 0.08 ). Left panel shows reflection coefficient variation for the dry case $(0 \%$ water saturation). Right panel shows the variation of reflection coefficients for the wet case (100\% water saturation). (b) Similar plot for higher fracture density of 0.16 .

b) Water saturation $0 \%$, high crack density

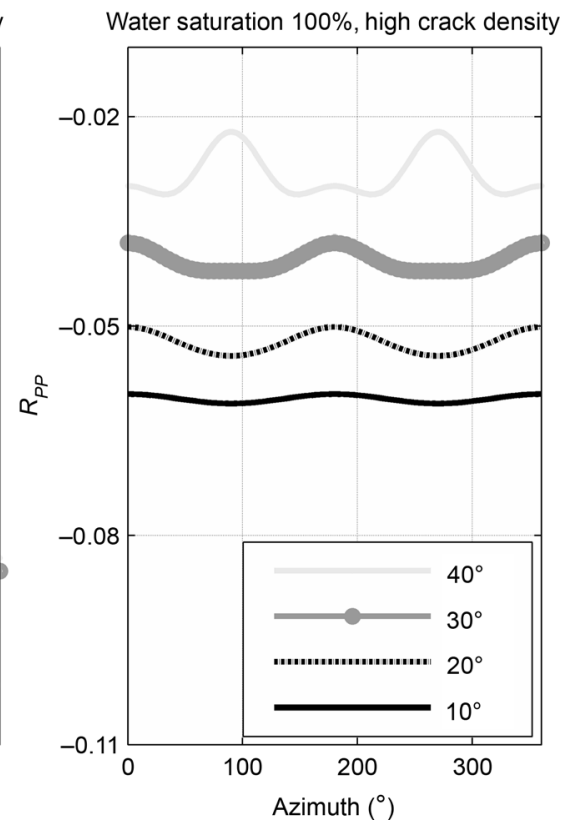


Table 2. Summary of influence of water saturation change $\left(\Delta S_{W}\right)$ from $0 \%$ to $100 \%$ on P-wave moduli $\left(\Delta C_{11}\right.$ and $\left.\Delta C_{33}\right)$, P-wave velocities $\left(\Delta V_{P}^{h}\right.$ and $\left.\Delta V_{P}^{v}\right)$, anisotropic parameters $\left(\Delta \gamma^{v}\right.$, $\Delta \varepsilon^{v}$, and, $\left.\Delta \delta^{v}\right)$, and anisotropic AVO gradient $\left(\Delta B_{A N I}\right)$

\begin{tabular}{lccccccccc} 
Case & $\Delta S_{W}$ & $\Delta C_{11}$ & $\Delta C_{33}$ & $\Delta V_{P}^{h}$ & $\Delta V_{P}^{v}$ & $\Delta \gamma^{v}$ & $\Delta \varepsilon^{v}$ & $\Delta \delta^{v}$ & $\Delta B_{A N I}$ \\
\hline 1 & $100 \%$ & $20 \%$ & $8 \%$ & $4.5 \%$ & $0.8 \%$ & $0 \%$ & $-20 \%$ & $0 \%$ & $100 \%$ \\
2 & $100 \%$ & $30 \%$ & $10 \%$ & $8 \%$ & $1.5 \%$ & $0 \%$ & $-21 \%$ & $1 \%$ & $100 \%$ \\
\hline
\end{tabular}

\section{CONCLUSIONS}

We perform limited sensitivity analysis of the porous vertically fractured anisotropic (HTI) medium fluid substitution equations. From our modeling, we observe that porosity and water saturation have a significant influence on the anisotropic properties of an HTI medium compared with that due to fracture density. An increase in porosity lowers the value of P-wave moduli and velocities. The decrement is more for the horizontal component than the vertical component. When fracture density is increased, we see an overall drop in the P-wave moduli and velocities (once again more drop in the horizontal component). However, the rate of change of P-wave moduli and velocities with porosity remains almost the same as those for the highand low-fracture-density case. Anisotropic parameters $\varepsilon^{v}$ and $\delta^{v}$ increase and decrease, respectively, with increasing porosity. We also observe that the anisotropic gradient increases significantly with an increase in porosity.

An increase in the water saturation causes an increase in the P-wave moduli and velocities of the porous HTI medium. The effect is more pronounced on the horizontal component than on the vertical component. The rate of change in the P-wave properties with water saturation is somewhat greater for the highfracture-density case but is not significantly different from the low-fracture-density case. Changes in the anisotropic parameters and anisotropic AVO gradients between the dry and wet states of an HTI medium are the same for the high- and low-fracturedensity case. Therefore, we conclude that water saturation may have a greater influence in seismic anisotropy than fracture density. We also observe that anisotropic AVO gradients increase between the dry and wet state of the porous HTI medium and the magnitude of the reflection coefficients decreases. In the future, similar work can be extended for the more complicated multiple fractured orthorhombic media.

\section{ACKNOWLEDGMENTS}

We thank ConocoPhillips for allowing us to present this work. Help from Tad Smith and intern Utpalendu Kuila is also appreciated. Amy Sharp from ConocoPhillips is acknowledged for editing the manuscript. Input from Tamas Nemeth, Marina Pervukhina, Jim Gaiser, and three other anonymous reviewers significantly improved the paper. Samik Sil thanks his wife Garima for constantly inspiring him to bring the paper to completion.

\section{REFERENCES}

Biot, M. A., and D. G. Willis, 1957, The elastic coefficients of the theory of consolidation: Journal of Applied Mechanics, 24, 594-601.

Brown, L., and B. Gurevich, 2004, Frequency dependent seismic anisotropy of porous rocks with penny-shaped cracks: Exploration Geophysics, 35, 111-115, doi:10.1071/EG04111.

Brown, R., and J. Korringa, 1975, On the dependence of the elastic properties of a porous rock on the compressibility of the pore fluid: Geophysics, 40, 608-616, doi:10.1190/1.1440551.

Cardona, R., 2002a, Two theories for fluid substitution in porous rocks with aligned cracks: 72nd Annual International Meeting, SEG, Expanded Abstracts, 173-176.

Cardona, R., 2002b, Fluid substitution theories and multicomponent seismic characterization of fractured reservoirs: Ph.D. thesis, Colorado School of Mines

Castagna, J. P., M. L. Batzle, and T. K. Kan, 1993, Rock physics - The link between rock properties and AVO response, in J. P. Castagna, and M. M Backus, eds., Offset-dependent reflectivity - Theory and practice of AVO analysis: SEG, 135-171.

Galvin, R. J., B. Gurevich, and C. M. Sayers, 2007, Fluid-dependent shear-wave splitting in a poroelastic medium with conjugate fracture sets: Geophysical Prospecting, 55, 333-343, doi:10.1111/j.13652478.2007.00593.x

Gassmann, F., 1951, Über die elastizität poröser medien: Vierteljahrschrift der Naturforschenden Gesellschaft in Zürich, 96, 1-23.

Gurevich, B., 2003, Elastic properties of saturated porous rocks with aligned fractures: Journal of Applied Geophysics, 54, 203-218, doi:10.1016/j.jappgeo.2002.11.002.

Gurevich, B., M. Brajanovski, R. J. Galvin, T. M. Müller, and J. TomsStewart, 2009, P-wave dispersion and attenuation in fractured and porous reservoirs - Poroelsticity approach: Geophysical Prospecting, 57, 225-237, doi:10.1111/j.1365-2478.2009.00785.x.

Hall, S. A., and J.-M. Kendall, 2000, Constraining the interpretation of AVOA for fracture characterisation, in Anisotropy 2000: Fractures, converted waves, and case studies: SEG, 107-144.

Holditch, S. A., 2006, Tight gas sands: Journal of Petroleum Technology, 58, 86-93, doi:10.2118/103356-MS

Hudson, J. A., T. Pointer, and E. Liu, 2001, Effective-medium theories for fluid-saturated materials with aligned cracks: Geophysical Prospecting, 49, no. 5, 509-522, doi:10.1046/j.1365-2478.2001.00272.x.

Mallick, S., K. Craft, L. Meister, and R. E. Chambers, 1998, Determination of the principal directions of azimuthal anisotropy from P-wave seismic data: Geophysics, 63, 692-706, doi:10.1190/1.1444369.

Mavko, G. M., T. Mukerji, and J. Dvorkin, 2003, The rock physics handbook, tools for seismic analysis in porous media: Cambridge University Press.

Nelson, R.A., 2001, Geologic analysis of naturally fractured reservoirs: Gulf Professional Publishing.

Neves, F. A., A. Al-Marzoug, J. J. Kim, and E. L. Nebrija, 2003, Fracture characterization of deep tight sands using azimuthal velocity and AVO seismic data in Saudi Arabia: The Leading Edge, 22, 469-475, doi:10.1190/1.1579581.

Rüger, A., 1997, P-wave reflection coefficients for transversely isotropic media with vertical and horizontal axis of symmetry: Geophysics, 62, 713-722, doi:10.1190/1.1444181.

Sayers, C. M., and M. Kachanov, 1995, Microcrack-induced elastic wave anisotropy of brittle rocks: Journal of Geophysical Research, 100, B3, 4149-4156, doi:10.1029/94JB03134.

Schoenberg, M. A., 2009, Vertically fractured transversely isotropic media: dimensionality and deconstruction: Geophysical Prospecting, 57, 169-185, doi:10.1111/j.1365-2478.2008.00775.x.

Schoenberg, M. A., and C. M. Sayers, 1995, Seismic anisotropy of fractured rock: Geophysics, 60, 204-211, doi:10.1190/1.1443748.

Sil, S., R. P. Srivastava, and M. K. Sen, 2010, Observation of shear-wave splitting in the multicomponent node data from Atlantis field, Gulf of Mexico: Geophysical Prospecting, 58, 953-964.

Smith, T. M., C. H. Sondergeld, and C. S. Rai, 2003, Gassmann fluid substitutions: A tutorial: Geophysics, 68, 430-440, doi:10.1190/1.1567211.

Thomsen, L., 1986, Weak elastic anisotropy: Geophysics, 51, 1954-1966, doi:10.1190/1.1442051.

Thomsen, L., 1995, Elastic anisotropy due to aligned cracks in porous rock: Geophysical Prospecting, 43, 805-829, doi:10.1111/j.13652478.1995.tb00282.x. 